\title{
Pointing sources for southern submm telescopes ${ }^{\star, \star \star}$
}

\author{
A. Adraou ${ }^{1}$, R. Chini ${ }^{1}$, M. Albrecht ${ }^{1}$, R. Lemke ${ }^{1}$, P. A. Shaver ${ }^{2}$, L.-Å. Nyman ${ }^{3}$, and R. S. Booth ${ }^{4}$ \\ 1 Astronomisches Institut der Ruhr-Universität Bochum, Universitätsstrasse 159/NA 7, 44780 Bochum, Germany \\ 2 European Southern Observatory, Karl-Schwarzschild-Strasse 2, 85748 Garching bei München, Germany \\ 3 Swedish-ESO Submillimetre Telescope, European Southern Observatory, Casilla 16001, Santiago 19, Chile \\ 4 Onsala Space Observatory, 43992 Onsala, Sweden
}

Received 23 April 2001 / Accepted 13 June 2001

\begin{abstract}
We present the results of a $230 \mathrm{GHz}$ survey of quasars and BL Lacertae objects performed with the SEST. The sample consists of objects with flat radio spectra and is taken from various radio surveys. Repeated observations for individual sources indicates variability by factors of $2-5$ within a single night. Comparing measurements over longer periods, even changes by factors of $10-25$ at $230 \mathrm{GHz}$ have been observed. The present catalog contains the 130 brightest objects whose $230 \mathrm{GHz}$ flux densities are above $500 \mathrm{mJy}$ most of the time. This, and their declination below $+20^{\circ}$, makes them useful candidates for future pointing sources at southern submillimeter telescopes. First measurements of flux densities at $345 \mathrm{GHz}$ are given for six objects.
\end{abstract}

Key words. galaxies: active - BL Lacertae objects: general - quasars: general - radio continuum: general - galaxies

\section{Introduction}

Bright compact submm sources with accurate positions are essential for future telescope projects such as: APEX (Atacama PAthfinder EXperiment) or ALMA in the southern hemisphere. Such sources not only will be required for the pointing accuracy of single dish telescopes but also for the phase calibration of interferometer arrays. Presently, the best way to find suitable objects is to observe bright flat-spectrum radio sources at $\mathrm{mm}$ wavelengths and to select those objects where the synchrotron emission has not yet turned down. Apart from their property of providing suitable calibration sources, these mm-bright sources belong to the most interesting active galactic nuclei and thus deserve intense study for their physical understanding. So far there exist two extensive mm-studies of southern radio sources: i) Beasley et al. (1997) observed flat-spectrum radio sources at 2 and $3 \mathrm{~mm}$ south of $-40^{\circ}$ and presented 52 objects which were detected at least at one wavelength above typical $5 \sigma$ limits of 300-500 mJy; ii) Tornikoski et al. (1996) monitored 230 and $115 \mathrm{GHz}$ flux densities of 155 extragalactic

Send offprint requests to: A. Adraou, e-mail: adraou@astro.ruhr-uni-bochum.de

* Based on observations obtained at the European Southern Observatory, La Silla, Chile.

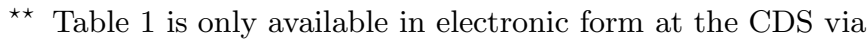
anonymous ftp to cdsarc.u-strasbg.fr (130.79.128.5) or via

http://cdsweb.u-strasbg.fr/cgi-bin/qcat?/A+A/376/1123 radio sources and give $230 \mathrm{GHz}$ data for 90 objects above the $500 \mathrm{mJy}$ level; most of these sources are in the declination range $-25^{\circ}$ to $+20^{\circ}$. Apart from these studies and neglecting single measurements on individual sources there exists no systematic search for (sub)mm-bright compact sources in the southern hemisphere. In the course of a larger survey on QSOs we have observed a large number of objects at $230 \mathrm{GHz}$. Simultaneously, we have started a similar survey at the HHT at a wavelength of $345 \mathrm{GHz}$. In this paper we present an extraction of the brightest QSOs and BLLacs from our $230 \mathrm{GHz}$ survey with $\delta<+20^{\circ}$ and $S_{230}>500 \mathrm{mJy}$; the catalog may be used to identify potential calibration candidates for southern submm telescopes. First results from our $345 \mathrm{GHz}$ survey are also included.

\section{The sample}

The sample consists of quasars and BL Lac objects, most of which were taken from the Parkes catalogue of radio sources (Shaver et al. 1996, 2001; Jackson et al. 2001; Hook et al. 2001). Included objects met the following criteria: (1) declination range $+2.5^{\circ}$ to $-80^{\circ}$ excluding low galactic latitudes $\left(|b|<10^{\circ}\right)$ and regions around the Magellanic Clouds; (2) flat spectral index between 2.7 and $4.85 \mathrm{GHz}$ $\left(\alpha>-0.4, S_{\nu} \propto \nu^{\alpha}\right)$; (3) $S>0.25 \mathrm{Jy}$ at $2.7 \mathrm{GHz}$.

In order to extend the spatial coverage at low declinations we searched in the Parkes-MIT-NRAO (PMN) Southern Survey $\left(-87.5^{\circ}<\delta<-37^{\circ}\right)$ for additional sources. This was done using the catalogue of Wright et al. (1997) who remeasured the strongest sources of the PMN 
Southern Survey (flux limits: $S_{4.85} \geq 50 \mathrm{mJy}\left(-87^{\circ}<\right.$ $\left.\left.\delta<-73^{\circ}\right), S_{4.85} \geq 70 \mathrm{mJy}\left(-73^{\circ}<\delta<-38.5^{\circ}\right)\right)$. We selected objects with an extrapolated flux density at $230 \mathrm{GHz}$ greater than $500 \mathrm{mJy}$. We also searched in the NASA/IPAC Extragalactic Database (NED) for additional southern radio sources. The selection criteria here were $\delta<-40^{\circ}$ and an extrapolated flux $S_{230} \geq 500 \mathrm{mJy}$. Obviously, the selection criterion of a flat spectral radio index is heavily influenced by the variability of the sources because in general observations at different frequencies were not performed simultaneously. Therefore it is likely that we have missed some stronger sources.

Finally, we included various objects with $\delta \leq+20^{\circ}$ that have been used by our group as pointing sources throughout the years and which had measured $230 \mathrm{GHz}$ flux densities greater than $500 \mathrm{mJy}$. Most of them were already available in the SEST Handbook (1999) or have been published elsewhere (Steppe et al. 1988, 1992, 1993, Reuter et al. 1997). In summary, we ended up with a sample of about 400 sources south of $\delta=+20^{\circ}$.

\section{Observations}

Apart from some older measurements from IRAM (30 m telescope) and SEST, most observations reported in this paper were carried out in 2000 during two periods, July 15 to 25 and November 3 to 12, at the SEST. The center frequency of both systems (IRAM and SEST) was $230 \mathrm{GHz}$ with a bandwidth of $60 \mathrm{GHz}$ at the SEST and $90 \mathrm{GHz}$ at IRAM. We used the single channel facility bolometer in the standard ON-OFF mode with a beam throw of $70^{\prime \prime}$. Calibration was done via skydips to determine the atmospheric transmission and by observing Uranus to obtain the absolute calibration. We estimate the calibration error to be about $10 \%$.

The $345 \mathrm{GHz}$ observations (bandwidth $=30 \mathrm{GHz}$ ) were performed in January 2001 at the HHT. We used the 19channel facility bolometer array in the standard ON-OFF mode with a beam throw of $70^{\prime \prime}$. Calibration was done as described above resulting in an uncertainty of about $20 \%$. In addition, we applied a skynoise reduction routine using the 18 surrounding channels of the array for baseline flattening.

\section{Results}

Alltogether, we observed 353 quasars and BL Lac objects at $230 \mathrm{GHz}$; the detection rate was $77 \%$ above the $3 \sigma$ limit. Due to the strong variability for many of the sources we selected only those objects as candidate pointing sources for future submm work which showed on average a $230 \mathrm{GHz}$ flux density above $500 \mathrm{mJy}$. Including a few previously measured QSOs - as mentioned above - we ended up with a catalog of $130 \mathrm{~mm}$-bright sources with $\delta \leq+20^{\circ}$ as given in Table 1

In Col. 1, the B1950 source name is given, Cols. 2 and 3 show the B1950 coordinates (see Jackson et al. 2001 for most of the sources). Column 4 contains the source type and Col. 5 gives the total number of observations including earlier measurements from IRAM and SEST. Most objects were observed several times and showed significant variability on short timescales (hours to days). Comparing our recent measurements with the data from the SEST and IRAM pointing catalogs (SEST Handbook 1999; Steppe 1988, 1992, 1993; Reuter 1997) we find $230 \mathrm{GHz}$ flux density changes between factors 3 and 25 . The signal-to-noise ratio of the quoted flux densities is at least five for the faintest objects and is significantly larger than ten for sources brighter than $1000 \mathrm{mJy}$. Detailed results on our variability studies will be presented in a separate paper (Adraou et al. 2001). In order to display the observed range of variability we give the minimum and maximum $230 \mathrm{GHz}$ values in Cols. 7 and 8 of Table 1, Col. 6 gives the average value. A few sources could be observed only once. To get an idea about their variability we included for comparison a second value from Tornikoski et al. (1996); these supplementary data from the literature are marked with an asterisk. Typically the average flux density of the objects is larger than $400 \mathrm{mJy}$, making them promising candidates for future submm pointing sources.

The spatial distribution of the objects is given in Fig. 1. While the coverage for $\delta>-60^{\circ}$ is fairly good the region $\delta<-60^{\circ}$ contains only 9 sources. Comparing the number of objects per square degree there are five times more sources in the region $\delta>-60^{\circ}$. This reflects the limited number of quasars known in the very south of the sky; additional work has to be done in this area.

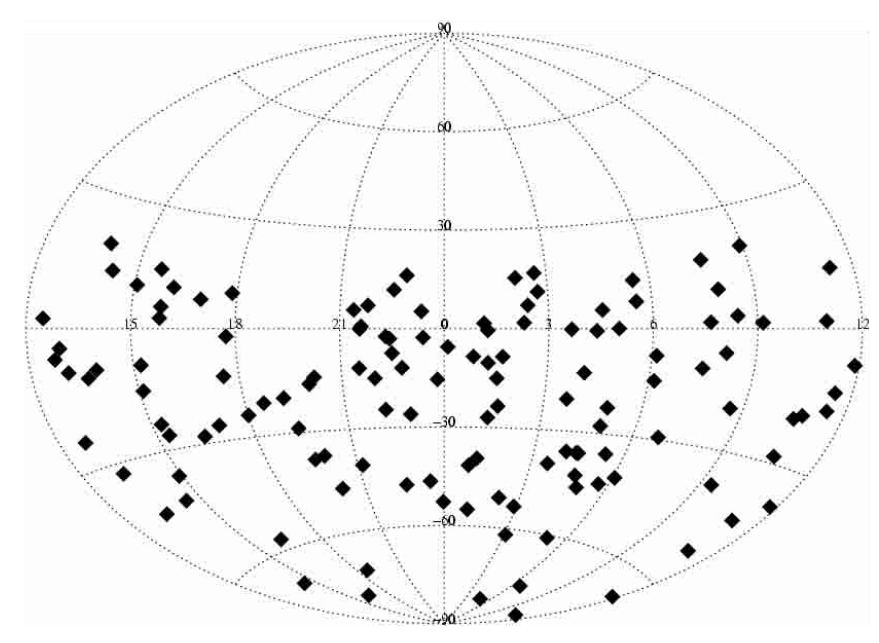

Fig. 1. Spatial distribution of the sources.

Table 2 gives the first results of our $345 \mathrm{GHz}$ survey. Although far from being representative, the small number of observed $345 \mathrm{GHz}$ flux densities lies well within the variability range of the $230 \mathrm{GHz}$ fluxes, suggesting that the synchrotron component remains rather flat even below $1 \mathrm{~mm}$. This corroborates the suitability of the sources from Table 1 as suited pointing sources for the submm regime. 
Table 2. Sources measured at $345 \mathrm{GHz}$.

\begin{tabular}{|c|c|c|}
\hline \multirow{2}{*}{$\begin{array}{l}\text { Name } \\
\text { B1655+077 }\end{array}$} & \multicolumn{2}{|c|}{$\begin{array}{c}S_{345} \mathrm{rms} \\
{[\mathrm{mJy}]}\end{array}$} \\
\hline & 508 & 56 \\
\hline B1749+096 & 2530 & 54 \\
\hline B2145+067 & 1189 & 43 \\
\hline B2223-052 & 4798 & 105 \\
\hline $\mathrm{B} 2230+114$ & 2230 & 77 \\
\hline B2251+158 & 2868 & 83 \\
\hline
\end{tabular}

\section{Conclusion}

From a total of about 400 quasars and BL Lac objects we have identified $130 \mathrm{~mm}$-bright sources suited for calibration purposes at future southern submm telescopes. All sources lie in the declination range from $+20^{\circ}$ to $-81^{\circ}$ and have $230 \mathrm{GHz}$ flux densities above $\sim 500$ mJy most of the time. Thus, the present sample is the most complete selection of strong $230 \mathrm{GHz}$ sources in the southern hemisphere.

Acknowledgements. We wish to thank C. Cesarsky for allocating part of her discretionary time for this project. It is a pleasure to thank the members of the SEST team for their continuous support during numerous observing runs. This work is based in part on measurements made with the Heinrich Hertz Telescope, which is operated by the Submillimeter Telescope Observatory on behalf of Steward Observatory and the Max-Planck-Institut fuer Radioastronomie. We want to thank R. Wielebinki and the HHT team for financial and technical support with the $345 \mathrm{GHz}$ observations. Special thanks go to
M. Nielbock for his help obtaining and reducing the $345 \mathrm{GHz}$ data. This research has made use of the NASA/IPAC Extragalactic Database (NED) which is operated by the Jet Propulsion Laboratory, California Institute of Technology, under contract with the National Aeronautics and Space Administration. Finally we want to thank our referee J. Bieging for numerous helpful comments.

\section{References}

Adraou, A., et al. 2001, in preparation

Beasley, A. J., Conway, J. E., Booth, R. S., Nyman, L.-Å., \& Holdaway, M. 1997, A\&AS, 124, 469

Booth, R. S., Delgado, G., Hagstrom, M., et al. 1989, A\&A, 216,315

Hook, I. M., et al. 2001, in preparation

Jackson, C. A., et al. 2001, in preparation

Reuter, H.-P., Kramer, C., Sievers, A., et al. 1997, A\&AS, 122, 271

SEST operating manual 1999, The SEST Handbook, Version 2.5

Shaver, P. A., Wall, J. V., Kellermann, K. I., Jackson, C. A., \& Hawkins, M. R. S. 1996, Nature, 384, 439

Shaver, P. A., et al. 2001, in preparation

Steppe, H., Salter, C. J., Chini, R., et al. 1988, A\&AS, 75, 317

Steppe, H., Liechti, S., Mauersberger, R., et al. 1992, A\&AS, 96, 441

Steppe, H., Paubert, G., Sievers, A., et al. 1993, A\&AS, 102, 611

Tornikoski, M., Valtaoja, E., Teräsranta, et al. 1996, A\&AS, 116,157

Wright, A. E., Tasker, N., McConnell, D., et al. 1997, Accurate positions, fluxes and structure for 6603 southern radio sources (Parkes internal data base) 